\title{
ON THE INTEGRATION OF THE EQUATION OF RADIATIVE TRANSFER
}

\author{
P. SWINGS AND L. DOR
}

\section{ABSTRACT}

The equation of the radiative transfer has been integrated by a generalization of Spitzer's method, which makes use of Bessel functions.

I. In solving the equation of radiative transfer appropriate to the problem of the formation of absorption lines, it is necessary to take into account the variation of the Planck intensity $B_{\nu}$ as function of the total optical depth within the line. To a sufficient approximation, $B_{\nu}$ may be expected to be a linear function of the form $a_{\nu}+b_{\nu} \tau$, where $\tau$ is the optical depth in the continuous spectrum; $\tau$ is defined with respect to the Rosseland mean absorption coefficient over the whole spectrum. On the other hand, we cannot, in general, expect $B_{\nu}$ to be a linear function of the optical depth $t_{\nu}$. The point at issue here is the circumstance to which Strömgren ${ }^{\mathrm{I}}$ has recently drawn attention:

Line absorption affects the intensity within the radiated spectrum in two ways. First, it cuts down the intensity as would increased continuous absorption, the effect depending upon the existence of a temperature gradient in the stellar atmosphere. Second, it causes a deviation from Kirchhoff's law, in the sense that emission is reduced, so that there is a further reduction in the radiated intensity.

Spitzer's recent solution ${ }^{2}$ of the equation of transfer in terms of Bessel functions is seen to take into account the second of the foregoing two effects "rigorously," while the first effect is taken into account by assuming for $B_{\nu}$ a linear function in $t_{\nu}$ (which assumption cannot generally be valid). For this reason the perturbation theory, developed by Strömgren, ${ }^{\mathrm{I}}$ is of greater value inasmuch as Strömgren's method in fact allows quite a considerable variation in $\eta_{\nu}{ }^{3}$

However, it is of some interest to supplement Spitzer's analysis

I $A p . J ., 86, \mathrm{I}, \mathrm{I} 937$.

2 Ap. J., 87, I, 1938 .

${ }^{3} A$ p. J., 86, I, I937, Table II.

$5^{16}$ 
by calling attention to the fact that several other possibilities of rigorous solutions exist for the equation of radiative transfer. Such possibilities are indicated here in a summarized form, no analytical discussion or expression of the residual intensity being added, as this would proceed along the same line as in Spitzer's paper. In any special example (i.e., for a definite model and frequency), it will be possible to select the analytical case which best approximates the physical conditions.

2. Using classical notations, the equation of radiative transfer may be written

$$
\frac{d^{2} J_{\nu}\left(t_{\nu}\right)}{d t_{\nu}^{2}}=3 \frac{\left(\mathrm{I}+\epsilon \eta_{\nu}\right)}{\mathrm{I}+\eta_{\nu}}\left\{J_{\nu}\left(t_{\nu}\right)-\frac{\mathrm{I}+\epsilon \eta_{\nu} Q}{\mathrm{I}+\epsilon \eta_{\nu}} B_{\nu}\left(t_{\nu}\right)\right\},
$$

where

$$
d t_{\nu}=\left(\mathrm{I}+\eta_{\nu}\right) d \tau_{\nu} .
$$

We assume, as usual,

$$
\frac{\mathrm{I}+\epsilon \eta_{\nu} Q}{\mathrm{I}+\epsilon \eta_{\nu}} B_{\nu}\left(t_{\nu}\right)=a_{\nu}+p_{\nu} t_{\nu}
$$

and substitute

$$
\begin{gathered}
y\left(t_{\nu}\right)=J_{\nu}\left(t_{\nu}\right)-\left(a_{\nu}+p_{\nu} t_{\nu}\right) \\
\lambda\left(t_{\nu}\right)=\frac{\mathrm{I}+\epsilon \eta_{\nu}}{\mathrm{I}+\eta_{\nu}}
\end{gathered}
$$

Thus, we get instead of (I)

$$
\frac{d^{2} y}{d t_{\nu}^{2}}=3^{\lambda}\left(t_{\nu}\right) \cdot y\left(t_{\nu}\right) .
$$

Spitzer's treatment consists essentially in finding adequate substitutions which convert equation (6) into a Bessel equation of the type

$$
\frac{d^{2} y}{d w^{2}}+\frac{\mathrm{I}}{w} \cdot \frac{d y}{d w}-\left(\mathrm{I}+\frac{n^{2}}{w^{2}}\right) y=0 .
$$


Solution I of Spitzer is

$$
\lambda=\frac{\mathrm{I}}{A}\left(\mathrm{I}+m t_{\nu}\right)^{-8 / 8+\mathrm{I}},
$$

with

$$
m=(s+\text { r }) \frac{D}{A},
$$

$s, A$, and $D$ being constants.

Solution II is

$$
\lambda=L+M e^{-u t_{\nu}},
$$

$L, M$, and $u$ being constants.

3. Substituting

$$
z=a+\beta t_{\nu},
$$

(6) keeps its essential form and becomes

$$
\frac{d^{2} y_{\mathrm{I}}}{d z^{2}}=\frac{3 \lambda(z)}{\beta^{2}} \cdot y_{\mathrm{I}}(z) .
$$

We may try to find a substitution of independent variable $w(z)$ and of unknown

$$
y(z)=u(z) \cdot y_{\mathrm{x}}(z),
$$

which transforms (7) into (9).

After some easy algebra, it is found that we need, therefore,

$$
\left\{\begin{array}{c}
\frac{d^{2} z}{d w^{2}}+\frac{\mathrm{I}}{w} \cdot \frac{d z}{d w}+\left(\frac{2}{u} \cdot \frac{d u}{d z}\right) \cdot\left(\frac{d z}{d w}\right)^{2}=0 \\
\left.-\frac{3 \lambda}{\beta^{2}}=\frac{\mathrm{I}}{u} \cdot \frac{d^{2} u}{d z^{2}}+\frac{\mathrm{I}}{\left(\frac{d z}{d u}\right)^{2}} \cdot\left[\frac{\mathrm{I}}{u} \cdot \frac{d u}{d z}\left(\frac{d^{2} z}{d w^{2}}+\frac{\mathrm{I}}{w} \frac{d z}{d w}\right)-\left(\mathrm{I}+\frac{n^{2}}{w^{2}}\right)\right]=0 . \text { (I I } 2\right)
\end{array}\right.
$$

Solution I of Spitzer corresponds to

$$
z=a w^{b} ; \quad u=z^{-\mathrm{I} / 2} ; \quad z=\mathrm{I}+m t_{\nu},
$$


$a, b$, and $m$ being constants which may be immediately related to Spitzer's coefficients.

Solution II corresponds to

$$
w=a e^{b z} ; \quad u=\text { const. } ; \quad z=t_{\nu} .
$$

The equations (II) and (I2) generalize Spitzer's results; (I I) may be replaced immediately by a first-order linear equation which brings

$$
\frac{d z}{d w}=\frac{\mathbf{I}}{w\left[\text { const. }+\int\left(\frac{2}{u} \cdot \frac{d u}{d z}\right) \cdot \frac{d w}{w}\right]} .
$$

We may take, for instance,

$$
u=e^{z} ; \quad z=a+b t_{\nu} ; \quad w=\left[\frac{\mathrm{I}}{A} \cdot e^{B e^{2(a+b t)}}\right]^{\mathrm{I} / 2}
$$

then $\lambda$ has the following form

$$
3^{\lambda}=b^{2}+b^{2} n^{2} B^{2} e^{4 z}+\frac{b^{2} B^{2}}{A} \cdot e^{4 z+B e^{2 z}}
$$

$a, b, A, B$, and $n$, being constants, and the solution is, accordingly,

$$
y=e^{-\left(a+b t_{\nu}\right)} \cdot\left[c_{\mathrm{I}} \cdot I_{n}(w)+c_{2} \cdot K_{n}(w)\right] .
$$

$I_{n}(w)$ and $K_{n}(w)$ are the usual Bessel functions of imaginary argument.

Obviously, instead of transforming (6) into (7), we could have taken the other Bessel equation, or, more generally, any other wellknown type of differential equation of the second order, namely, that providing the orthogonal polynomials.

4. Equation (6) being linear and homogeneous with regard to $y$ and $d^{2} y / d t^{2}$, the substitution

$$
y=e^{\int u d t}
$$


will transform (6) into a Riccati equation

$$
\frac{d u}{d t}+u^{2}=3^{\lambda}(t)
$$

and a solution by quadratures is always obtainable when

$$
3 \lambda(t)=\frac{d \mu}{d t}+\mu^{2}
$$

$\mu$ being any function of $t$.

The solution is actually

$$
u=\mu-\frac{\mathrm{I}}{v}
$$

with

$$
v=e^{2} \int_{\mu d t}\left\{c_{\mathrm{I}}-\int e^{-2} \int_{\mu d t} d t\right\}
$$

For instance, if

$$
\mu=\frac{f^{\prime}(t)}{f(t)} \quad \text { or } \quad 3 \lambda(t)=\frac{f^{\prime \prime}(t)}{f(t)}
$$

$f(t)$ being any function, we have

$$
y=c_{\mathrm{x}} f(t)+c_{2} f(t) \cdot \int \frac{d t}{f^{2}(t)}
$$

The simple case $f(t)=A t^{m}$ brings

$$
{ }_{3} \lambda(t)=\frac{m(m-\mathrm{I})}{t^{2}} \text { and } y=c_{\mathrm{I}} t^{m}+c_{2} t^{-m+\mathrm{x}} .
$$

5. Let

$$
y=f(t)
$$

be a solution of (6); suppose we may write $t=F(y)$. Then (6) becomes

$$
\frac{d^{2} y}{d t^{2}}=3 \lambda[F(y)] \cdot y=\varphi(y) \cdot y
$$


Thus

$$
d t=\left[2 \int \varphi(y) \cdot y \cdot d y+c_{\mathrm{I}}\right]^{-1 / 2} \cdot d y .
$$

By quadrature we shall find $t$ as a function of $y$.

In other words, if we take any function $\varphi(y)$, we shall get $y=f(t)$ by quadrature, and then

$$
3^{\lambda}(t)=\varphi(y)=\varphi[f(t)] .
$$

For instance, if

$$
\varphi(y)=\frac{A}{y}
$$

we find

$$
y=\frac{A}{2} t^{2}+B t+C
$$

with

$$
3^{\lambda}=\frac{2 A}{A t^{2}+2 B t+2 C},
$$

$A, B$, and $C$ being constants.

6. Summarizing, we may state: (I) Spitzer's method introducing Bessel functions may be generalized by formulae (II) and (I2) [Example: formula (I5)]; (2) and substitution (I6) give a solution by quadrature when $\lambda(t)$ verifies the general relation (I7) [Examples: formulae (2O) and (2I)]; (3) another integration by quadrature is given by formula (26); this may in some cases give simple solutions.

Our thanks are due to S. Chandrasekhar for valuable suggestions.

UNIVERSITY OF LIEGE, BELGIUM

DEPARTMENT OF ASTROPHYSICS April ro, 1938 